\title{
Una aproximación teórica a las representaciones sociales y su relación con los estudiantes*
}

\author{
Lilaitzel Carrasco Rodríguez \\ Universidad Popular Autónoma del Estado de Puebla \\ Correo electrónico: lilaitzel.carrasco@gmail.com
}

Recibido: 25 de septiembre de 2015 - aprobado:30 de octubre de 2015

\section{Cómo citar este artículo}

Carrasco, L. (2015). Una aproximación teórica a las representaciones sociales y su relación con los estudiantes. Espiral. Revista de Docencia e Investigación, 5(2), 97-110.

\section{Resumen}

El presente artículo es una revisión bibliográfica, construido a partir de la lectura de artículos, libros y trabajos publicados de manera reciente. El objetivo central es profundizar el conocimiento sobre el tema de las "representaciones sociales de los estudiantes de educación media superior, su relación con la universidad y el vínculo que existe entre estas y educación". En ese sentido, el artículo proporciona información para la reflexión y entendimiento de las representaciones sociales, a partir de uno de los principales actores en el sistema educativo que son los estudiantes. También la investigación representa un esfuerzo por concentrar algunas de los principales estudios sobre dichos temas.

La metodología empleada fue la siguiente: primero, definir el tema por investigar, posteriormente, la búsqueda de información y revisión de literatura en redes de revistas especializadas con las siguientes características: artículos de años recientes, todos publicados en revistas especializadas en educación. El tercer paso fue la organización de la información por categorías y, por último, el análisis de las investigaciones. En las conclusiones se muestra que más que conceptos contrapuestos, la educación y las representaciones sociales se relacionan para la construcción de nuevos conocimientos sobre los imaginarios, ideales, expectativas que poseen los estudiantes y cómo es que lo representan. Los autores que se consultaron como base para esta investigación fueron Serge Moscovici y Denise Jodelet.

Palabras clave: Educación, educación media superior, estudiantes, representaciones sociales, universidad.

\section{Abstract}

This article is a literature review, constructed from reading articles, books and papers recently published. The main objective is to deepen the state of knowledge on the subject of the students' Social Representations of in upper secondary education, their relationship with the university and the link between Social Representations and Education. In that sense, this paper provides information for reflection and understanding of the social representations from one of the main characters in the education systems, which are the students.

The research also represents an effort to concentrate some of the major research on these issues, the methodology used was as follows: First, the research topic was defined, then finding information and literature review journals networks with the following characteristics: recent years articles, all of them published in education journals. The third step was the organization of information by categories and finally the analysis of the research. The conclusions show that more than competing concepts, education and social representations are related to the construction of new knowledge about imaginaries, ideals, expectations that students have and how they are represented. The authors used as a basis for this research were Serge Moscovici and Denise Jodelet.

Keywords: Education, upper higher education, social representations, university.

\section{Introducción}

La actividad constante de investigación es un medio esencial para la profundización de un tema en específico y este trabajo pretende alcanzar lo anterior; por tanto, la finalidad de esta revisión bibliográfica es ampliar el conocimiento para los estudiosos de las Representaciones

* Artículo de revisión bibliográfica: que hace parte del proyecto de tesis doctoral en Pedagogía, que la autora adelanta en la Universidad Popular Autónoma del Estado de Puebla (UPAEP). 
Sociales y las investigaciones recientes sobre dicho tema y la educación. También es resultado de una búsqueda que tiene como finalidad mostrar una parte del estado del conocimiento sobre el tema de las representaciones sociales y la relación que guarda entre las representaciones y la educación, posteriormente, las representaciones y los estudiantes de educación media superior y la relación con la universidad. Los criterios utilizados para tal revisión fueron: estudios con información reciente, pertinente y cercana a los estudiantes de educación, se realizó una búsqueda en Internet comprobando que cada uno de los artículos revisados hubiese sido publicado en revistas especializadas con arbitraje. De esta información se dio preferencia a los escritos publicados que trabajaron concretamente con las representaciones sociales, de investigadores de reconocido prestigio y que tomaron como base la teoría propuesta por Serge Moscovici, también se buscó que los investigadores trabajaran en instituciones de investigación o educación.

De la información presentada en el apartado de resultados, se encuentran tres grupos y se ordenaron de la siguiente manera, primero, los estudios que abordan las representaciones sociales y la educación para aportar una perspectiva más general sobre el tema y la relación entre ambos campos, después, los trabajos sobre representaciones sociales y los estudiantes de educación superior $y$, al final, los relacionados con las representaciones sociales y la educación media superior (EMS). Hay que mencionar que no todos los trabajos analizados fueron elaborados para México, pero resultan importantes para estudiar lo que ocurre en otras partes relacionado con el estado de conocimiento en representaciones sociales. Posteriormente. un espacio de conclusiones y para finalizar las referencias.

\section{Metodología}

La revisión bibliográfica constituye un elemento importante en la investigación científi- ca, ya que marca los nuevos tópicos estudiados o, bien, las posibles líneas por investigar sobre un tema determinado. La metodología que de manera frecuente se propone para la revisión bibliográfica muestra la posibilidad de ser utilizada en cualquier tema de investigación y sirve para determinar la preeminencia e importancia de este. Igualmente permite que otros investigadores consulten las fuentes bibliográficas citadas, para un conocimiento más profundo, mejorar la comprensión y ahondar sobre el tema. La metodología utilizada para esta revisión bibliográfica fue la propuesta por Fernando-Navas y Gómez-Luna (2014) y se utilizaron cuatro pasos esenciales en la revisión de bibliografía sobre el tema de representaciones sociales. La primera fase para la revisión bibliográfica fue la definición del problema, este se pensó que debía ser claro y específico para realizar una búsqueda bibliográfica de calidad y que sobre todo respondiera a la necesidad de conocer sobre el estado del arte de las representaciones sociales y los estudiantes. La finalidad de la delimitación del problema fue construir un espacio de investigación lo suficientemente amplio para dar paso a la crítica y reflexión. La segunda etapa que se utilizó para esta revisión fue la búsqueda de información, sobre todo en fuentes confiables y de alta calidad, con reconocimiento en la comunidad científica, la búsqueda fue en redes de revistas indexadas, con arbitraje académico, en un primer momento que abordaran el tema de las representaciones sociales y la educación sin importar el nivel o el enfoque dado a estas. También se consultaron algunas tesis doctorales sobre el tema central y alguna relación con un ámbito escolar. Un espacio extra de consulta fueron diversas memorias de congresos sobre educación y representaciones sociales. Los libros de autores clásicos como Serge Moscovici y Denise Jodelet y Abric marcaron una visión holística y compleja sobre cómo abordar las representaciones.

Posterior a la búsqueda de información, se procedió a la organización de la pesquisa, se or- 
denó de manera sistemática y así se construyeron las categorías que se presentan en la sección de resultados. La forma en que se jerarquizó la información fue de la siguiente manera: primero los artículos que tuvieran una relación entre representaciones sociales y educación, después, investigaciones que trabajaron representaciones sociales con estudiantes universitarios $y$, por último, con estudiantes de educación media superior. La última etapa fue la más compleja, ya que se determinaron los documentos de mayor utilidad para la revisión bibliográfica, los que se ven reflejados y analizados en el siguiente apartado, fue la etapa a la que se dedicó más tiempo, pero tuvo resultados provechosos, útiles y valiosos para la investigación.

Los frutos de la metodología descrita anteriormente son los que a continuación se describen, en primer lugar, investigaciones que se han enfocado en explicar, relacionar y fortalecer la línea que une a las representaciones sociales con la educación. Después algunos estudios que analizaron diversas formas de representaciones en varios temas con universitarios, sin importar la carrera, semestre o universidad $y$, para cerrar, aportaciones que refieren a los estudiantes de educación media superior y las diversas representaciones sociales que abordan en situaciones particulares.

\section{Resultados}

Durante la revisión bibliográfica sobre representaciones sociales y educación, se observaron algunas tendencias, particularidades y espacios en los cuales no se ha trabajado con profundidad el tema. Las tendencias hacen referencia a mostrar el lenguaje como un medio eficaz y necesario para la construcción de la realidad, que a su vez se ve reflejada en las representaciones sociales de los estudiantes y de algunos más que hacen su participación en el proceso educativo.

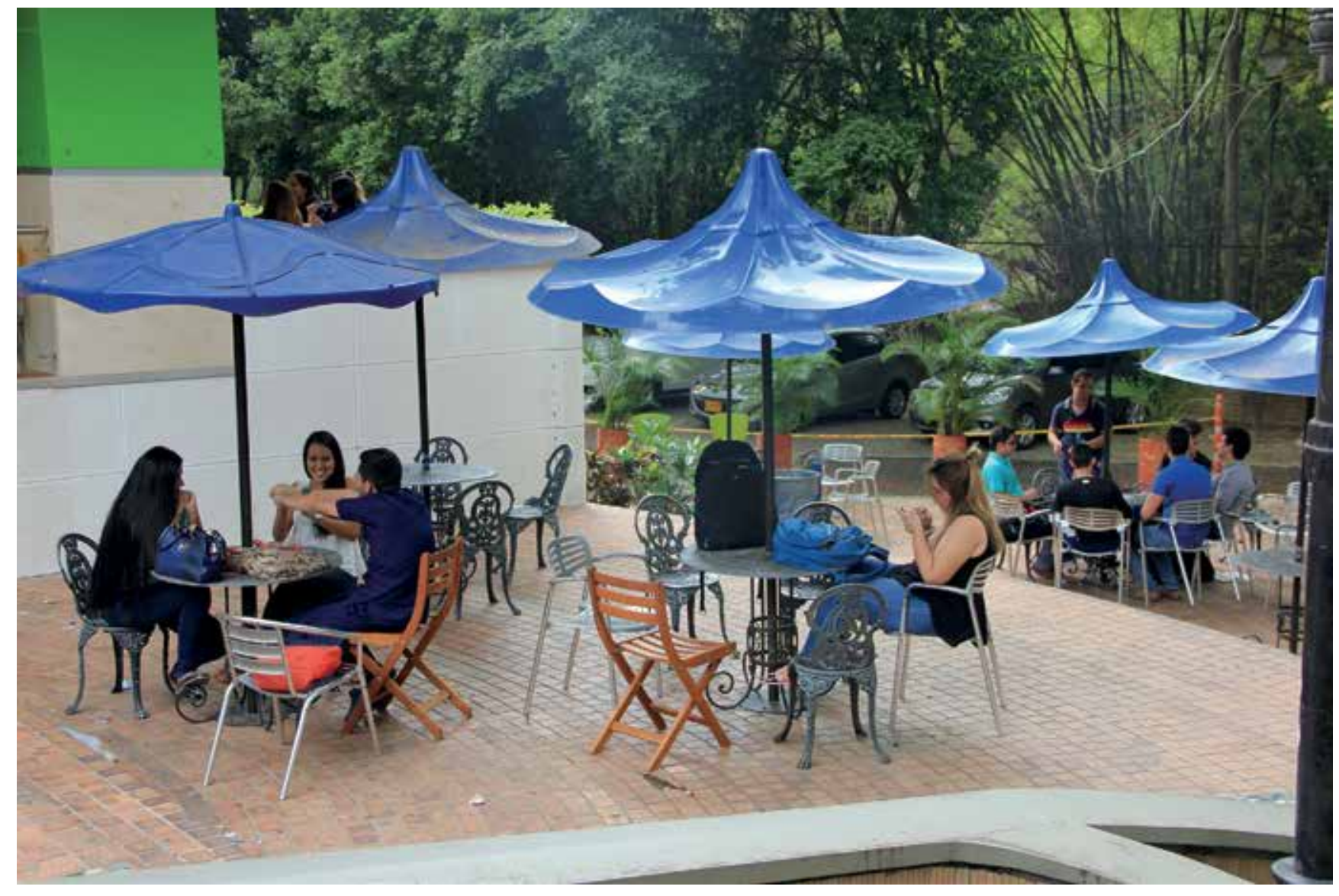


La metodología empleada es similar en todas los estudios revisados en este trabajo, la finalidad es observar y analizar con detenimiento las respuestas, categorías y construcciones de los involucrados y la obtención de la información más precisa, conociendo los proceso que los llevan a esas determinadas representaciones sociales.

En todo momento, es importante tener claro la relación que existe entre las representaciones sociales y la educación, ambas se nutren y complementan sus campos, por ello y con base en la revisión bibliográfica realizada, se construyeron tres espacios de estudios que evidencian cómo las representaciones sociales están presentes en todo el proceso de enseñanzaaprendizaje.

\section{Estudios que se caracterizan por analizar a las representaciones sociales y su relación con la educación}

Las investigaciones que evidencian tal vínculo son completamente teóricas, no hay investigaciones de campo ni resultados de dichos trabajos, es decir, son elaboraciones teóricas y meramente conceptuales, únicamente se abordan las relaciones establecidas entre las representaciones sociales y el campo de la educación. Algunos de los estudios son: Vergara (2008) quien trabajó un artículo llamado "La naturaleza de las representaciones Sociales, este estudio aborda a las representaciones sociales como una especie de guía de acción y marco para entender la realidad. Basándose en las aportaciones teóricas de Moscovici, explica la autora que las representaciones sociales son necesarias e importantes para abordar los problemas de la sociedad. Las representaciones sociales permiten explicar el curso de los sucesos cotidianos y las actuaciones sociales; pronuncian las relaciones que las personas mantienen con el mundo y con los otros. Explica la manera en que las representaciones sociales se manifiestan en y con el lenguaje y en las prácticas, en razón de su función simbólica y de los referentes que proporcionan para codificar y categorizar el mundo, la aproximación a las representaciones sociales se constituye en un aparato teórico completo para profundizar en el conocimiento de las concepciones y prácticas que orientan la experiencia de vida de los diferentes grupos poblacionales. El artículo solo recoge las orientaciones teóricas y no hace aproximaciones metodológicas.

El estudio llamado "Aportes del enfoque de las representaciones sociales al campo de la educación" de Denis Jodelet (2011) examina las relaciones entre la educación y las representaciones sociales con el propósito de mostrar la pertinencia de los recursos que ofrece el estudio de estas últimas para el análisis de los procesos que se ponen en juego en los diferentes niveles del sistema educativo. Luego de poner en evidencia los lazos históricos existentes entre los dos campos, la autora insiste sobre la necesidad de preservar su especificidad. La autora despeja las líneas directrices según las cuales los dos campos de estudio se reencuentran. Las representaciones sociales dan testimonio de una evolución de las funciones conferidas a la educación y a la escuela, y afectan las concepciones de los roles y oficios de los docentes y de los alumnos. El rol decisivo que desempeñan en la relación entre los distintos actores del sistema educativo es ilustrado con diversos ejemplos.

Un aporte más de las representaciones a la educación como lo menciona Jodelet es que orientan el funcionamiento del sistema escolar en materia de organización de los conocimientos y los saberes útiles a la producción. El modelo al que obedece el sistema puede ser considerado como una representación jerarquizada de saberes, según un orden unidimensional que ubica en la cima el saber científico, que hace del saber técnico la aplicación de este último y que tiene él mismo como aplicación el saber profesional. Esta representación tiene muchas implicancias a nivel de la imagen de las 
formaciones, de la construcción de cursos y de la representación de los formados.

Una investigación teórica que abunda sobre representaciones sociales y educación es la de Piña y Cuevas (2003) "La teoría de las representaciones sociales. Su uso en la investigación educativa en México", menciona que en México, durante los últimos años, se han llevado a cabo investigaciones que tienen como objeto de estudio las representaciones sociales de los agentes educativos: estudiantes, profesores, autoridades y padres de familia. Las representaciones sociales las configura un sujeto o grupos de sujetos y se refieren a algo o a alguien: una institución, un contenido, una metodología, un acontecimiento, un reglamento, entre otros.

El propósito de este trabajo consiste en hacer un esbozo de la teoría de las representaciones sociales a partir de las propuestas de sus principales exponentes y analizar las modalidades metodológicas que esta propuesta teórica ha tenido en la investigación educativa en México. Un objetivo más de este trabajo consistió en destacar la importancia del pensamiento de sentido común para la investigación educativa en Méxi$\mathrm{co}$, se analizaron las modalidades que adquiere la producción en representaciones sociales en el campo educativo en nuestro país y, por último, algunas consideraciones generales.

Otro artículo que señala la relación entre las representaciones sociales y la educación es el referido a "Representaciones sociales: Una manera de entender las ideas de nuestros alumnos" de Lacolla (2005). Se estudia en una primera parte a las representaciones sociales: una intersección entre la ciencia y la sociedad, en el que trata de clarificar el concepto y en tanto que las representaciones sociales pueden ser consideradas como procesos y productos. Después aborda las características de las representaciones sociales y también la conformación de una representación social, con dos procesos denominados: mecanismos de objetivación y mecanismos de anclaje; por último, explica el estudio desarrollado además de las representaciones sociales y las actitudes de sus alumnos hacia la ciencia.

Es importante el abordaje de las representaciones sociales y la relación estrecha que existe con el campo de la educación, se complementan y han sido estudiadas desde años atrás para conocer, determinar y reflexionar sobre las representaciones sociales y cómo estas influyen en los procesos educativos.

La segunda categoría construida hace referencia a.

\section{Estudios sobre representaciones sociales y su relación con la educación superior}

Estas son investigaciones que se refieren concretamente al ámbito universitario y estudios sobre representaciones sociales y su relación con estudiantes universitarios, algunos de los avances de investigación fueron los siguientes:

El trabajo llamado "Representaciones sociales sobre inclusión de personas con discapacidad en educación superior" de Isaacs y Mansilla (2014). El presente estudio tuvo como propósito determinar y describir las representaciones sociales de estudiantes universitarios de la ciudad de Temuco sobre inclusión y discapacidad. Se empleó la técnica de Redes Semánticas Naturales. La muestra estuvo conformada por 20 estudiantes universitarios pertenecientes a distintas instituciones de educación superior de Temuco, se empleó un muestreo no probabilístico intencional para la recogida de los datos. La edad de los participantes oscila entre los 18 y 25 años, entre los cuales se contemplan 14 mujeres y 6 hombres.

Los análisis se realizaron con base en los siguientes valores establecidos a partir del desarrollo de la técnica utilizada: Valor J: número total de palabras definidoras, representa la riqueza semántica de la red; valor M: producto de la frecuencia de un nodo por su valor se- 
mántico, indica el peso semántico de cada palabra definidora; conjunto SAM: compuesto por las quince palabras definidoras de mayor valor $M$, ordenadas de menor a mayor, indica las definidoras fundamentales en la red de un grupo; valor FMG: este valor se obtiene para cada una de las palabras del conjunto SAM, a través de la regla de tres simple, tomando como punto de partida que la palabra definidora con el valor M mayor, representa el $100 \%$. Este valor es un indicador en términos porcentuales de la distancia semántica existente entre las diferentes palabras que conformaron el conjunto SAM. Los valores arrojados fueron los siguientes: Inclusión (66), Discapacidad (55), Inclusión en educación superior (70) y Discapacidad en educación superior (50). Con objeto de analizar los datos obtenidos a partir del muestreo.

Las representaciones sociales están presentes todo el tiempo en los procesos educativos y sobre todo en los estudiantes, ya que participan en la construcción de estas en comunión y comunicación con el resto de sus compañeros y al llegar a la universidad son una parte fundamental para el aprendizaje, así, el artículo llamado "Representaciones de estudiantes universitarios sobre el aprendizaje significativo y las condiciones que lo favorecen" de Covarrubias y Martínez (2007) analiza las representaciones y los significados que estudiantes universitarios asignan al aprendizaje y a las condiciones que lo promueven en el interior del salón de clases. Para tal situación, las autoras utilizaron una metodología de corte cualitativa-interpretativa que reivindica al sujeto como constructor de la realidad, permite obtener su subjetividad e identificar sus principales representaciones (Covarrubias y Martínez, 2007). Las investigadoras determinaron que la participación debería ser de 25 estudiantes que se encontraban cursando alguno de los dos últimos semestres de la carrera de Psicología de la Facultad de Estudios Superiores (FES), Iztacala, de la Universidad Nacional Autónoma de México (UNAM), con la finalidad de que pudieran relatar y expli- car sus experiencias académicas a lo largo de su trayectoria universitaria. Las autoras decidieron trabajar con una entrevista semiestructurada, ya que lo consideraron un instrumento flexible. En tal instrumento se construyeron las preguntas guías y rectoras para la investigación: ¿qué entienden los estudiantes por "aprender"?, ¿qué significado otorgan al aprendizaje y cómo suponen que se logra?, ¿qué condiciones prefieren para el aprendizaje?, ¿cómo perciben su propio aprendizaje de acuerdo con sus experiencias? Los resultados obtenidos a los cuestionamientos anteriores les permitieron a las autoras establecer las representaciones sociales de los estudiantes, de la siguiente manera: a) conceptualización del aprendizaje, b) técnicas didácticas que auxilian el aprendizaje, c) funciones de los profesores que favorecen el aprendizaje significativo, d) actitudes de los profesores que favorecen el aprendizaje significativo, e) valoración del propio aprendizaje (Covarrubias y Martínez, 2007).

La escuela al ser un espacio fértil de convivencia, formación y movilidad, se comparten y transforman saberes, opiniones y percepciones, la escuela funciona como un receptor de Representaciones Sociales ya que es el lugar en el que se comparten ideas, costumbres, experiencias, se refuerzan algunas y otras más se transforman, es así que el artículo "Representaciones acerca de la pobreza, desigualdad social y movilidad socioeconómica en estudiantes universitarios de la ciudad de Barranquilla, Colombia" de Amar, Abello, Denegri, Llanos, Pardo, Villadiego, Florez y Duque (2006), indagó las representaciones sociales que construyen los jóvenes entre los 19 y los 24 años de edad de una institución universitaria de la ciudad de Barranquilla en torno a los fenómenos de pobreza, desigualdad social y movilidad socioeconómica. La población con la que se trabajó en este estudio estuvo conformada por 50 estudiantes, entre los 19 y 24 años de edad, seleccionados de forma intencional. Estos se encontraban matriculados en los programas de Dere- 


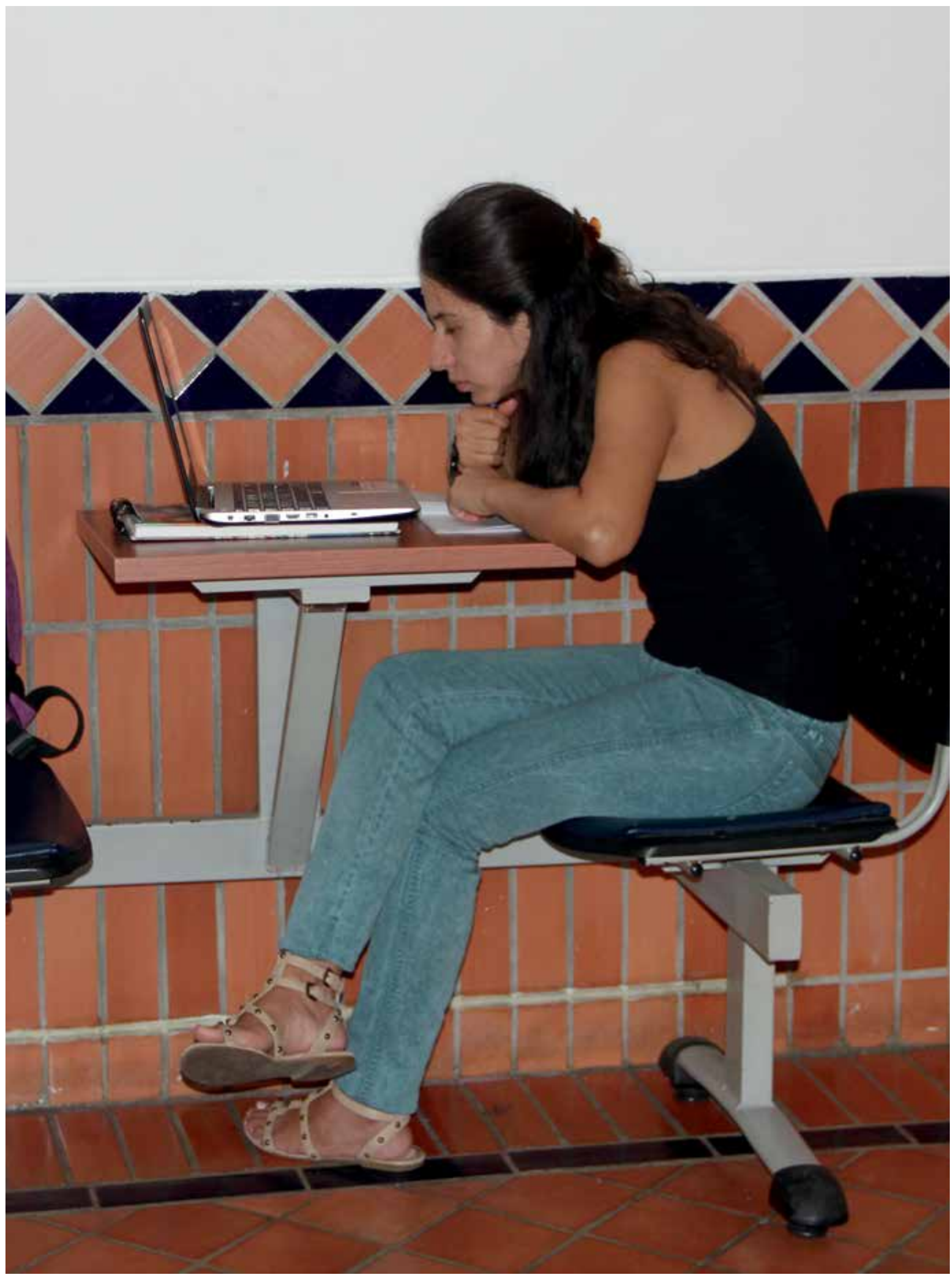


cho, Psicología, Ingeniería Industrial, Medicina y Administración de Empresas, pertenecientes a los niveles socioeconómicos alto y medio. Se les aplicó la entrevista clínica individual piagetiana en profundidad, de carácter semiestructurada, la cual se encuentra dividida en tres apartados: el primero se refiere a la caracterización de ricos y pobres, el segundo, al proceso de movilidad social, y el tercero hace referencia a las soluciones al problema de la pobreza. Se establecieron cuatro niveles de pensamiento sobre las representaciones sociales acerca de la pobreza y la desigualdad social. Estos niveles de pensamiento reflejan el contenido y la estructura de las representaciones de los sujetos; por tal motivo se presentan a continuación los porcentajes en los que se ubican los sujetos de la presente investigación con respecto a dichos niveles. Los investigadores mencionan que esperaban que los sujetos analizados alcanzaran el nivel de pensamiento IV, pese a esto, solo un $10 \%$, es decir, es el nivel más alto de comprensión de la estructura social y es el peldaño en el que hay un menor número de estudiantes. Un porcentaje significativo de sujetos (62\%) se ubicó en el nivel III de pensamiento, este se caracteriza por una comprensión inicial de la movilidad socio-económica como un proceso que se da por etapas y un porcentaje (28\%) de jóvenes ubicados en el nivel II de pensamiento. Los individuos que se encuentran en este nivel se centran en los elementos más llamativos y perceptibles a la vez que presentan concepciones poco realistas basadas en su imaginación, medios de comunicación o experiencias de otros compañeros para elaborar sus explicaciones. $Y$ el nivel I tiene $0 \%$ de estudiantes.

Existen diferentes representaciones sociales para un solo tema, estas dependen de quién las aborde y la finalidad de estas, así el estudio llamado "Representaciones sociales sobre la escritura de la tesis en la formación académica inicial en el área de las Ciencias del Mar" de Tapia y Marinkovich (2011) las dos autoras de universidades chilenas, abordaron el estudio de las representaciones sociales y ratificaron la importancia de que en la culminación de la formación universitaria es necesario realizar una tarea de escritura, esta tarea es la llamada tesis. Las representaciones que sobre la tesis tienen tanto estudiantes como profesores son resultado de sus propias experiencias y expectativas sociales y académicas. El objetivo de este artículo es mostrar las representaciones que un grupo de docentes y de estudiantes de dos carreras diferentes evidencian con respecto a lo que es la escritura, en especial a la "tesis". Después del trabajo realizado se obtuvo información acerca de cómo representan los docentes y estudiantes la escritura de la tesis, qué relación existe entre esa representación y los pasos de la investigación científica y qué función social cumple en el ámbito académico. Los resultados revelan la diferente concepción que sobre la tesis tienen docentes y estudiantes. Los primeros representan la tarea como parte de un eslabón que debe concretarse en un artículo científico publicable que favorezca el ingreso a estudios de posgrado y los estudiantes perciben la tesis como una tarea difícil de desarrollar, pues consideran que no fueron lo suficientemente preparados para enfrentarla. Las representaciones parecen mantenerse en distintos supuestos: la tesis como un medio para cerrar una etapa de formación académica e ingresar al mundo laboral y la tesis como un medio para acceder a los estudios de posgrado. La investigación empleó una metodología de carácter cualitativo-interpretativa y se escogió el enfoque metodológico denominado Teoría Fundamentada (TF). La muestra inicial se conformó a partir de 12 casos entre profesores y estudiantes del último año del área de las Ciencias del Mar (Química Marina y Biología Marina) de la Universidad Católica de la Santísima de la ciudad de Concepción. Estos casos cumplieron con los criterios de inclusión para el estudio: a) profesores que guían tesis de pregrado y b) estudiantes que escribieron una tesis de pregrado. Se comentan los resultados en función del interrogante central de la investigación, cuál es, 
cómo se representan socialmente las tesis de pregrado profesores y alumnos de dos carreras asociadas a las Ciencias del Mar. Las representaciones sociales de los estudiantes mostraron que conciben la escritura y elaboración de la tesis como un requisito por cumplir para obtener el grado académico, mientras que los docentes representan la escritura de la tesis como una posibilidad para acceder a mejor estudios de posgrado (Tapia Ladino, 2011).

Las representaciones sociales abordadas desde la educación superior arrojan datos interesantes y se construyen categorías útiles para su análisis, la universidad es un espacio necesario de convivencia y transformación de las representaciones sociales.

Las representaciones sociales y su relación con la educación abordan una línea interesante de estudio, las representaciones no se construyen aisladas sino en comunión y la escuela por tanto es un espacio fértil para ellas. Es necesario conocer cuáles son las representaciones sociales de los estudiantes en el nivel medio superior, porque supone un momento determinante para ellos porque marca un proceso decisivo en su futuro académico y en el nivel superior porque es probable que las representaciones construidas durante sus vidas hayan cambiado como parte de la mediación con el mundo.

\section{Estudios sobre representaciones sociales y el estudiante de educación media superior}

Estos artículos abordan el estudio de las representaciones sociales y su relación con determinadas áreas del conocimiento y tienen la particularidad de que se enfocaron en estudiantes de educación media superior.

El artículo de Martínez (2011) habla sobre las representaciones sociales, percepciones, ideas e imágenes que del aprendizaje y enseñanza de las matemáticas tiene un grupo de estudiantes del nivel medio superior del área de Física y Matemáticas del Instituto Politécnico Nacional. La investigación fue de tipo cualitativo, la metodología de la investigación se apoyó en cuestionario y entrevistas realizadas en grupos focales. El objetivo fue generar discursos escritos y hablados que permitieran conocer la representación social. En cada sesión trabajaron de doce a quince estudiantes y dos entrevistadores, ninguno era profesor de los estudiantes. Los pasos para trabajar fueron los siguientes: 1) aplicación del cuestionario de manera individual; 2) conformación en grupos de tres o cuatro estudiantes de acuerdo con sus preferencias; 3 ) responder el cuestionario de manera colectiva; 4) comentar y precisar las respuestas con los entrevistadores (Martínez Sierra, 2011). Para tener una mayor certeza sobre las respuestas emitidas por los estudiantes, los investigadores en las etapas siguientes, segunda, tercera y cuarta, decidieron hacer un trabajo de grabación en dos vertientes, la primera en audio y la otra en imágenes. El autor decidió trabajar con 67 estudiantes de quinto semestre de una escuela orientada al área de Física. Los resultados señalados por Martínez muestran lo siguiente: se identificaron dimensiones que permiten conocer la organización de los contenidos en las representaciones. Tales dimensiones se construyeron a partir de las interpretaciones que los jóvenes hicieron de las preguntas abiertas, que de manera sintética se ha identificado con otra pregunta. A su vez, en cada dimensión se identificaron categorías que se presentaron a través de frases que tienen por objetivo reconstruir el significado global del tipo de respuesta dada por los estudiantes. Se construyeron dimensiones que permiten conocer la organización de los contenidos en las representaciones. En cada dimensión se construyeron categorías que se presentaron a través de frases que tienen por objetivo reconstruir el significado global del tipo de respuesta dada por los estudiantes (Martínez, 2011).

Si los docentes y directivos tuvieran un conocimiento más especializado sobre lo que los estudiantes piensan, sienten y conciben sus representaciones sociales sobre la escuela po- 
drían atacar directamente los problemas educativos, sobre esta línea, un estudio más se refiere a la tesis de doctorado llamada "Representaciones sociales de las carreras universitarias de estudiantes de tercer año de una preparatoria oficial" de Barberena (2008), que tenía como objetivo conocer las representaciones sociales de los estudiantes de último año de bachillerato sobre las carreras universitarias ofrecidas por una Universidad Tecnológica. Para realizar el estudio, la tesis se dividió en seis apartados, los dos primeros hacen referencia a investigaciones teóricas sobre el tema de representaciones sociales, la orientación educativa y el proceso de toma de decisiones, también hace referencia a la influencia de la familia, el contexto social y económico que rodea a los estudiantes. En la parte tres, se habla del contexto donde se realizó la investigación, los antecedentes de las universidades tecnológicas y las carreras de técnico superior universitario. En el siguiente capítulo se trabaja la metodología por seguir para el trabajo de campo, en el quinto capítulo se muestran los resultados y, por último, las conclusiones. Los resultados fueron categorizados en tres apartados; el primero: los estudiantes consideran a la Universidad Tecnológica como una "buena escuela", segundo apartado se relaciona con las expectativas de los alumnos y las carreras que ofrece la Universidad y el último fue la comparación entre los alumnos de sexto semestre con respecto a la Universidad Tecnológica de Tulancingo. Las representaciones sociales tienden a una cuestión cultural y existen patrones comunes entre los estudiantes.

En este bloque de análisis se identifica que la finalidad primordial de las investigaciones es conocer las representaciones sociales de los adolescentes en la etapa de educación media superior, es decir, el imaginario, lo que piensan y cómo conciben en partícular alguna materia determinada, y sobre la elección de las diversas carreras universitarias; es importante conocer cómo es que los bachilleres determinan sus representaciones sociales.

\section{Conclusiones}

La teoría de las representaciones sociales fue elaborada y desarrollada por Serge Moscovici, muestra una complejidad que pretende estudiar las formas de pensar, actuar y las costumbres, ideas, imaginarios y construcciones mentales de individuos o de grupos sociales que muestran cómo diversos elementos participan en la formación de dichas representaciones.

Las representaciones sociales tienen su orígen en la psicología social, y aunque fueron elaboradas en un campo distinto al de la educación, la relación entre las representaciones y la educación fue una preocupación constante para Moscovici y a la que dedicó una parte importante de sus investigaciones para construir tal vínculo y que posteriormente Denise Jodelet reforzó.

Una tendencia en la primera categoria analizada es la necesidad de mostrar la relación existente entre las representaciones sociales y educación, no importa el nivel educativo o el área específica de estudio, es relevante dejar en claro que son conceptos que se complementan para ampliar el objeto de estudio de uno o del otro. Por lo tanto es importante saber cómo se construyen las representaciones sociales desde los estudiantes.

Los estudios sobre representaciones sociales y educación en cualquier nivel son determinantes para conocer los imaginarios, expectativas, deseos y esperanzas que tienen los estudiantes sobre sus procesos educativos.

Un elemento central que todas las categorías toman en cuenta, sin considerar el nivel educativo, es la importancia que tiene el lenguaje para los dos campos estudiados, las representaciones sociales y la educación; la trascendencia del lenguaje en estos ámbitos es que sirve para aprender a nombrar la realidad, tanto de las representaciones como de la educación. El lenguaje será uno de los elementos constitutivos que sirve de base para poder relacionar 
ambos campos sin perder la línea conductora, es decir, el lenguaje construye un puente que evidencia la fortaleza del vínculo entre las representaciones y el ámbito educativo.

Un hueco que se encontró en los diversos estudios de representaciones sociales y la educación, es que se han centrado en estudiantes de educación secundaria, media superior, superior y posgrado, por lo que los estudios en niveles inferiores son complicados de encontrar.

Las representaciones sociales no están aisladas de procesos académicos, culturales, inclusivos ni de cualquier índole y en la universidad el sujeto toma nuevas ideas, reconfigura otras y construye realidades antes no pensadas, se puede decir que los estudiantes universitarios edifican nuevas representaciones y las comparten con sus pares, cambian el pensar que aprendieron en casa y socializan el nuevo. También las representaciones ayudan a que cada estudiante pueda asignar significados concretos al aprendizaje y a las condiciones que lo propician.

Las representaciones sociales y sus estudios se han empeñado en mostrar lo útiles que son estas para la sociedad, no son elementos aislados ni construidos superficialmente, sino que se encuentran inmersas en todas las situaciones de la vida. Además de la utilidad en lo cotidiano, las representaciones sociales también muestran un rigor científico para su estudio.

No es posible ni correcto pensar que solo hay un tipo o categoría de representación, hay diversidad de ellas, depende de quien las aborde, puede ser el mismo tema pero diferente representación, y ahí se encuentra una de las principales riquezas de este tema, polisemia en la construcción y entendimiento de estas formas de conocimiento.

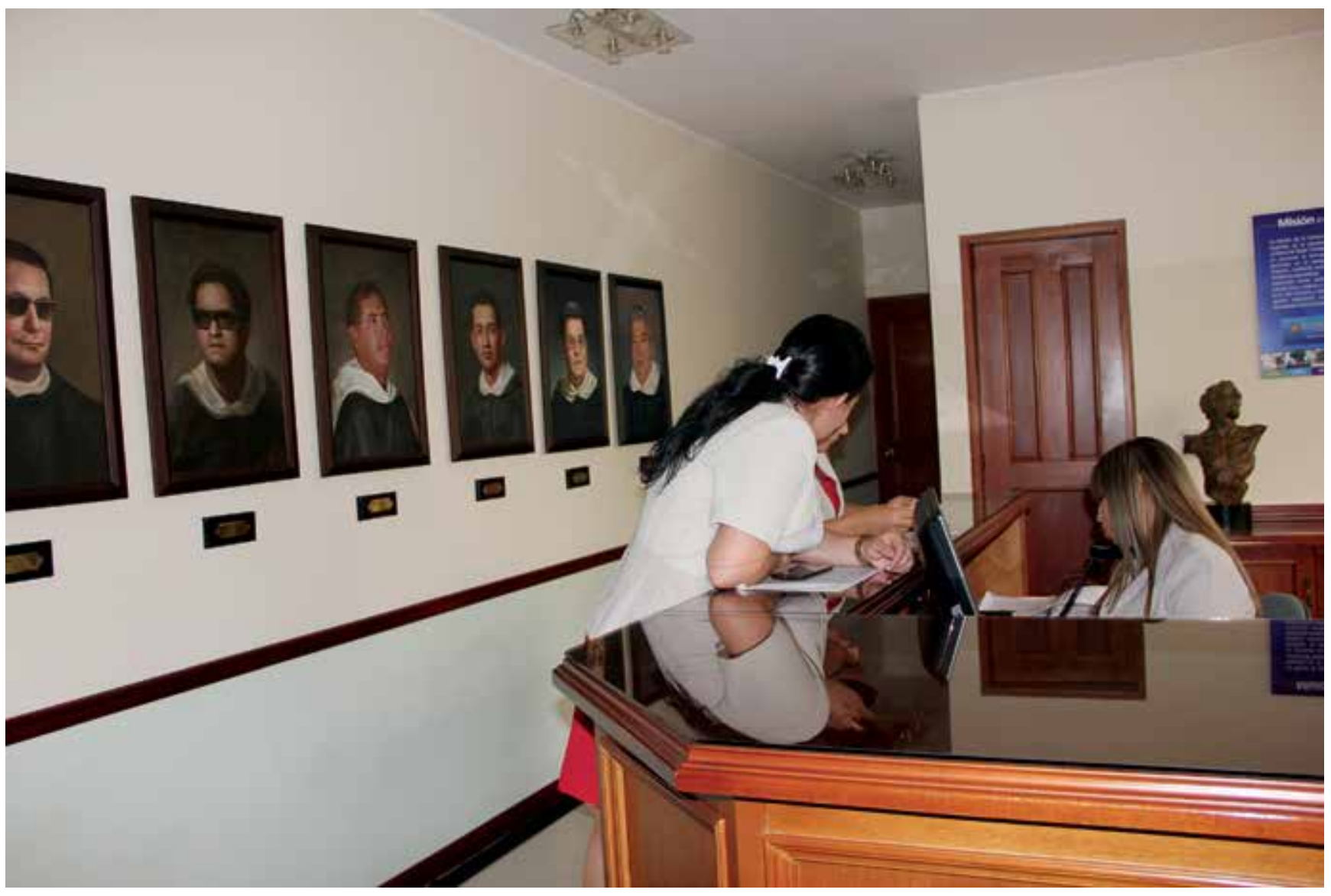




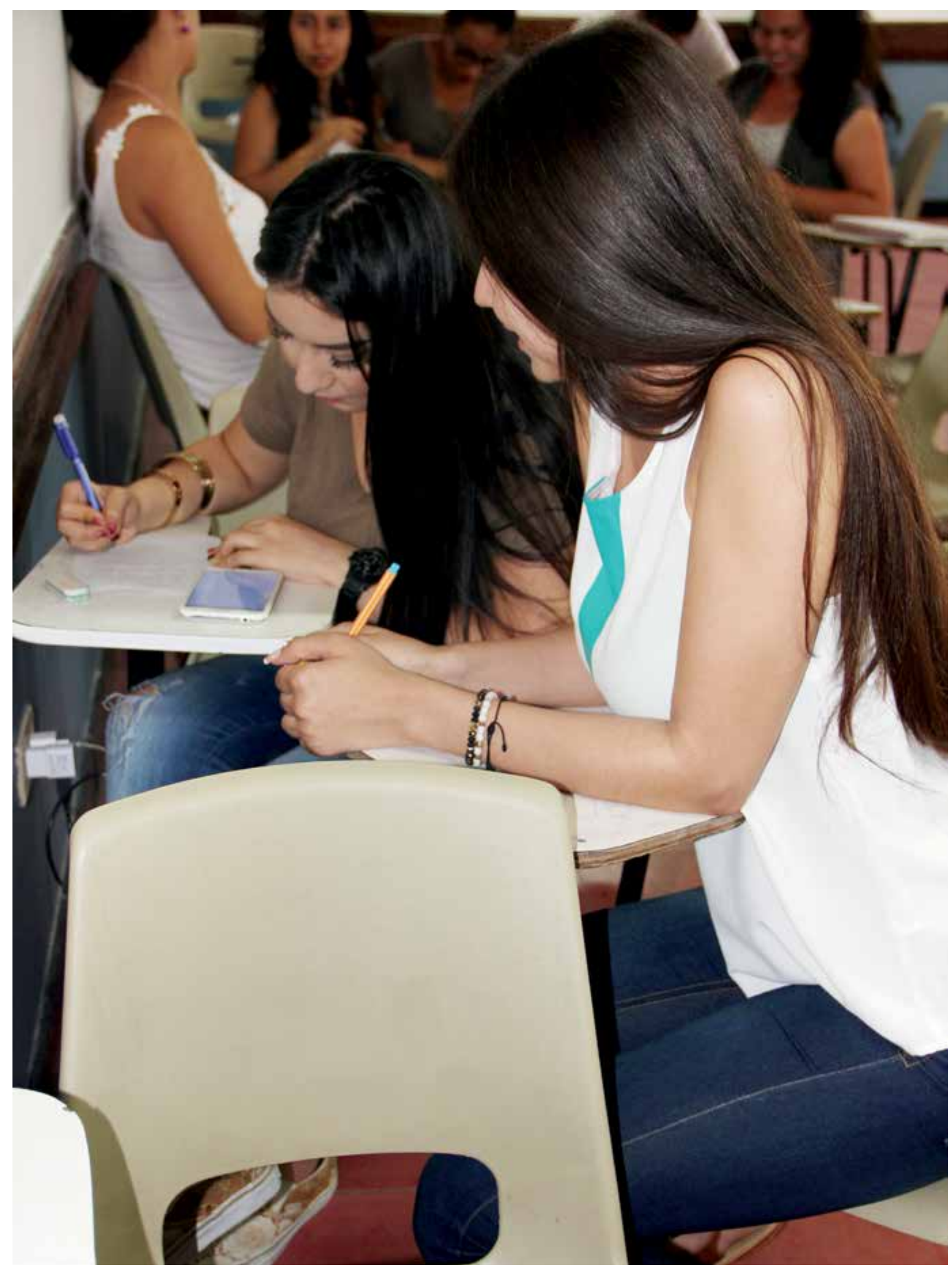


Una tendencia más encontrada en la revisión bibliográfica de las tres categorías es que se utiliza una metodología de tipo cualitativo, con entrevistas a profundidad, grupos focales, redes semánticas o charlas directas con los involucrados. Se podría pensar que una finalidad de esta metodologia empleada es mostrar que los estudiantes son los constructores de su realidad y, por tanto, de sus propias representaciones, pero sin perder de vista la relación con otros factores.

La educación media superior es vista como un paso determinante para la construcción del futuro, mismo que puede ser laboral o académico, en este nivel es trascendental lo que los estudiantes piensan y sienten sobre sus propios procesos educativos, ya sea como escuela, asignatura o área específica. Las escuelas, secundarias, bachilleres o universitarias deben preocuparse por conocer, analizar y determinar las representaciones sociales de sus estudiantes, atenderlas, transformarlas si es necesario, repensarlas y construir para mejorar los procesos educativos.

Una relación entre las últimas dos categorías es la de tomar en cuenta las expectativas, experiencias e imaginarios en los diversos estudios entre representaciones y educación supeior y media superior. De la misma manera, en los últimos dos análisis se busca comparar o contrastar dos o más categorías, grupos, características o algún otro elemento que tenga como finalidad la contemplación de la diversidad de representaciones sociales.

Una ausencia detectada en los análisis revisados es que no contemplan las representaciones sociales en cuanto al desempeño académico de estudiantes con altas y bajas calificaciones y lo que ello repercute en sus decisiones futuras sobre la universidad. Es decir, no toman en cuenta el paso fundamental que representa cambiar de un nivel educativo a otro ni las consecuencias que conllevan sus decisiones.
Las representaciones sociales y la educación son conceptos que se pueden enlazar y apoyar para edificar nuevas y mejores relaciones sociales y reivindicar los procesos educativos para la formación de seres críticos, reflexivos, propositivos y conscientes.

\section{Referencias}

Abric, J. (2004). Metodología de recolección de las representaciones sociales. En Abric, J. Prácticas sociales y representaciones (pp. 53-74). México: Ediciones Coyoacán.

Amar, J., Abello, R., Denegri, M., Llanos, M., Pardo, M., ViIladiego, T., Florez, C., \& Duque, S. (2006). Representaciones acerca de la pobreza, desigualdad social y movilidad socioeconómica en estudiantes universitarios de la ciudad de Barranquilla, Colombia. Investigación \& Desarrollo, 14(2) 312-329.

Barberena, D. (2008). Las representaciones sociales de los alumnos de sexto semestre de bachillerato sobre las carreras de Técnico Superior Universitario en la Universidad Tecnológica de Tulancingo y su repercusión en la matrícula. (Tesis de doctorado). Universidad Autónoma del Estado de Hidalgo.

Berger, P. (1991). La construcción social de la realidad. Buenos Aires: Amorrortu.

Bruner, J. (1984). Acción, pensamiento y lenguaje. Madrid: Alianza.

Cominetti, R., Ruiz, G. (1997). Algunos factores del rendimiento: las expectativas y el género. Human Development Department. LCSHD Paper series, 20. The World Bank, Latin America and Caribbean Regional Office.

Covarrubias, P. (2011). Representaciones sobre la enseñanza. Una indagación en estudiantes universitarios. Sinéctica, (36), 1-18.

Covarrubias, P., y Martínez, C. (2007). Representaciones de estudiantes universitarios sobre el aprendizaje significativo y las condiciones que lo favorecen. Perfiles Educativos, XXIX(115), 49-71.

Covarrubias, P., y Piña, M. (2004). La interacción maestroalumno y su relación con el aprendizaje. Revista Latinoamericana de Estudios Educativos, XXXIV(1), 47-84.

Covarrubias, P., y Tovar, L. (2005). Las representaciones de los estudiantes sobre la evaluación de su aprendiza- 
je. En M. A. Campos. (Coord.). Construcción de conocimiento en el proceso educativo. México: CESU-UNAM/ Plaza y Valdez.

Flores, M. (2015). Representaciones sociales de la Licenciatura en Trabajo Social: una perspectiva de los alumnos que solicitan cambio de carrera. Congreso Internacional de Educación. Currículum 2015.

Isaacs, M., y Mansilla, L. (2014). Representaciones Sociales sobre inclusión de personas con discapacidad en educación superior. REXE. Revista de Estudios y Experiencias en Educación, 13(26), 117-130.

Jiménez, M. (2000). Competencia social: intervención preventiva en la escuela. Infancia y Sociedad. 24, 21-48.

Jodelet, D. (1986). La representación social: Fenómenos, conceptos y teoría. En Moscovici, S. "Psicología social II'. Pensamiento y vida social. Psicología social y problemas sociales (pp. 469-494). Barcelona: Paidós.

Jodelet, D. (2000). Representaciones sociales: contribución a un saber sociocultural sin frontera. En Jodelet, D., y Guerrero, A. (Coords.). Develando la cultura. Estudios en representaciones sociales (pp. 7-30). Ciudad de México: UNAM.

Jodelet, D. (2004). Experiencia y representaciones sociales. En Romero, E. (Ed.). Representaciones sociales. Atisbos y cavilaciones del devenir de cuatro décadas (pp. 85-118). Puebla: BUAP.

Jodelet, D. (2011). Aportes del enfoque de las representaciones sociales al campo de la educación. Espacios en blanco. Serie Indagaciones, 21(1), 133-154.

Lacolla, L. (2005). Representaciones sociales: una manera de entender las ideas de nuestros alumnos. Revista Electrónica de la Red de Investigación Educativa, 1(3).
Fernando-Navas, D; Betancourt-Buitrago, L; Gómez-Luna, E; Aponte-Mayor, G; (2014). Metodología para la revisión bibliográfica y la gestión de información de temas científicos, a través de su estructuración y sistematización. Dyna, (81) 158-163.

Martínez, G. (2011). Representaciones sociales que poseen estudiantes de nivel medio superior acerca del aprendizaje y enseñanza de las matemáticas. Perfiles Educativos, XXXIII(132), 90-109.

Moscovici, S. (1979). El psicoanálisis, su imagen y su público. Buenos Aires: Heumul.

Moscovici, S. (1981). Psicología de las minorías activas. España: Morata.

Moscovici, S. (1984). Psicología social II. España: Paidós.

Moscovici, S., y Marková, I. (2003). La presentación de las representaciones sociales: diálogo con Serge Moscovici. En Castorina, J. A. (Comp.). Representaciones sociales. Problemas teóricos y conocimientos infantiles (pp. 111-151). Barcelona: Gedisa.

Piña, J., \& Cuevas, Y. (2004). La teoría de las representaciones sociales. Su uso en la investigación educativa en México. Perfiles Educativos, XXVI(106), 102-124.

Tapia, M., y Marinkovich, J. (2011). Representaciones sociales sobre la escritura de la tesis en la formación académica inicial en el área de las Ciencias del Mar. Onomázein, 2(24), 273-297.

Vergara, M. (2008). La naturaleza de las representaciones sociales. Revista Latinoamericana de Ciencias Sociales, Niñez y Juventud, 6(1), 55-80. 